Int. J. Dev. Biol. 50: 659-663 (2006)

doi: $10.1387 / \mathrm{ijdb} .062186 \mathrm{as}$

Meeting Report

\title{
Eye development at the Houston "Fly Meeting"
}

\author{
AMIT SINGH* \\ Department of Molecular and Cellular Biology, Baylor College of Medicine, Houston, Texas, USA
}

\begin{abstract}
The 47th Annual Drosophila Research Conference or "Fly Meeting" took place at Houston, Texas, USA from March 29th- April 2nd, 2006, under the aegis of the Genetics Society of America. The Fly Meeting provides an excellent opportunity for fly researchers to present their work and to get a snapshot of recent developments and upcoming trends in their research field. The fruit fly, Drosophila melanogaster is a very versatile model to study growth, patterning, neural development, evolution, systemetics and various other facets of biomedical science. The topics presented in the meeting covered a very broad spectrum of fly research. In this commentary, I have focused mainly on the presentations related to two fields: 1) research in various fields that use the Drosophila eye as a model system, and 2) the community resources available to all fly researchers.
\end{abstract}

KEY WORDS: Drosophila melanogaster, patterning, development, eye

The Genetics Society of America (GSA) organizes an annual meeting for Drosophila researchers from all over the world who work on overlapping or closely related fields, for interaction and exchange of ideas. The $47^{\text {th }}$ Annual DrosophilaResearch Conference was organized at Houston, Texas, USA by Hugo J. Bellen, Ron Davis, Graeme Mardon (Baylor College of Medicine) and Georg Halder (M.D. Anderson Cancer Research Center). The meeting had an attendance of nearly 1,500 people and had 18 platform sessions encompassing 136 talks, 13 workshops and about 1,000 posters.

The meeting began with a keynote address by Thomas Kaufman from Indiana University Bloomington. His talk entitled " $A$ century of Drosophila: 100 years of counting" focused on major milestones of Drosophila research along the time axis of the last 100 years and his research on the Antennapedia gene complex in flies. It was followed by a talk from Daniel Ortiz-Barrientos (Indiana University, Bloomington, USA), the winner of the Professor Larry Sandler memorial lecture award for his $\mathrm{PhD}$ dissertation work on molecular genetics of speciation. He used Drosophila pseudoobscura as a model system of speciation to investigate how speciation occurs in the face of gene flow.

The first plenary session covered many diverse fields such as plasticity in pheromone-driven courtship behavior in flies ( $L$. Griffith, Brandeis University, Waltham, MA, USA), regulation of planar cell polarity through Frizzled signaling (M. Mlodzik, Mount Sinai School of Medicine, New York, USA), progress on the sequencing of the heterochromatin region of the Drosophila genome (G. Karpen, University of California, Berkeley, CA, USA), mechanism of odor-perception in flies through a battery of odorreceptors (L. Vosshall, The Rockfeller University, New York, USA) and generation of morphological diversity during evolution by changes in cis-regulatory sequences of genes (S. Carroll, University of Wisconsin, Madison, USA).

Sean Carroll gave a talk entitled "Endless flies most beautiful: diversity through cis-regulatory evolution"about the evolution of morphologically similar traits in different Drosophila species. Carroll and colleagues studied the evolution of wing pigmentation pattern in flies in various Drosophilidae species. Interestingly, changes in a few nucleotides in the cis-regulatory element of the yellow pigmentation gene were responsible for the generation of different wing spot patterns in related species. He suggested that the modular cis-regulatory elements can be regulated to generate diversity and novelty during evolution and morphologically similar traits can evolve independently. These studies are important because they provide critical evidence for the evolution of adaptive traits by modulation of regulatory elements. He concluded his talk with a wonderful movie reminding us of the many important contributions made by naturalists and evolutionary biologists.

The topics presented in the meeting covered a wide spectrum of fly research. In this commentary, I will focus on presentations

Abbreviations used in this paper: Dan, distal antenna gene; DV, dorso-ventral; GSA, Genetics Society of America; IOB, intraommatidial bristles; NMNAT, nocotinamide mononucleotide adenyltransferase; PCP, planar cell polarity; $\mathrm{RD}$, retinal determination; Slp, sloppy paired gene; SMW, second mitotic wave; Std, stardust gene. 
related to the Drosophila eye and the recent additions to the community resources.

Despite the morphological diversity in the eyes of different organisms, the basic genetic machinery required for visual system development is highly conserved. In Drosophila, the progenitors for all adult epidermal structures are present as a group of cells inside the larva called imaginal discs. The eye imaginal discs arise from 20 cells set aside at the end of embryonic development, which develop into the adult compound eye of Drosophila comprising of nearly 800 unit eyes or ommatidia. The adult compound eye and head structures of the fly develop from eye imaginal discs (Fig. 1), which undergo complex signaling events controlling growth. The Drosophila eye with its unique structure and flawless design is a powerful model system for studying the molecular and genetic mechanisms involved in cell fate specification and differentiation. The fly eye is also an excellent model organ to assay defects in tissue growth, planar cell polarity and to model several human diseases.

\section{Cell polarity}

The Drosophilaeye is a highly organized neurocrystalline lattice consisting of ommatidia and serves as an excellent model to study planar cell polarity (PCP). Each ommatidium or the unit eye is made up of eight photoreceptor cells. PCP is manifested in the arrangement of the photoreceptor cells in the ommatidial clusters. PCP is generated during the third larval instar eye imaginal disc.

In the first plenary session, Marek Mlodzik (Mount Sinai School of Medicine, New York, USA) presented recent findings from his group on the "Regulation and specificity of Frizzled/ Planar Cell Polarity signaling." The establishment of cellular polarity is a crucial step in the development of epithelial tissues. Marek elaborated on the role of a newly identified gene, diego, in organizing the planar polarity of Drosophila photoreceptor neurons and hair orientation in wing cells. Diego is an ankyrin-repeat protein that localizes to the distal membrane where Frizzled and Dishevelled

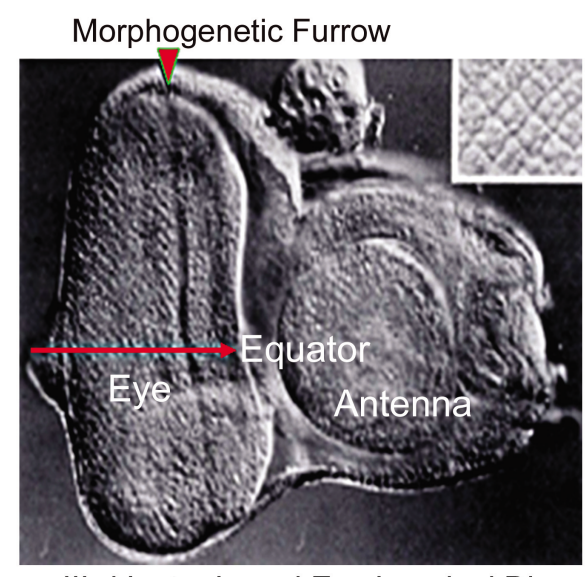

IIIrd Instar Larval Eye Imaginal Disc

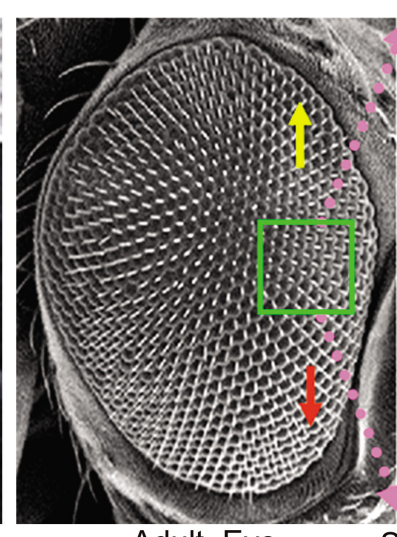

Adult Eye
Fig. 1. The larval eye imaginal disc develops into the compound eye and head of the adult fly. The compound eye of the adult fly comprises about 800 ommatidia (photoreceptor clusters), which are arranged with mirror-image symmetry (as shown in the section of the eye) along the dorsal and ventral axis. Each ommatidium comprises eight photoreceptors arranged in a neurocrystalline lattice. Courtesy of Dr. K. Choi.

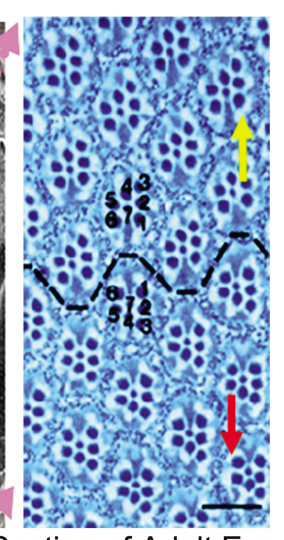

Section of Adult Eye are localized. Diego directly binds Dishevelled and promotes Frizzled signaling. Diego promotes hyper-phosphorylation of Dishevelled and this interaction is antagonized by Prickle. Deigo and Prickle compete for binding with Dishevelled. Thus, Diego modulates Frizzled/ Dishevelled activity to control Frizzled planar cell polarity signaling. Marek's group has also carried out an RNAi based screen for kinases and phosphatases involved in planar cell polarity and found some interesting candidates [e.g. Par1, casein kinase I epsilon $(\mathrm{CKI})$ )] that will help to decipher how Frizzled/ Dishevelled mediated signaling differs from Wingless signaling.

In order to understand the mechanism of generation of PCP in the eye, it is essential to identify the genetic circuitry involved in this process. Domingos et al. talked about a new role for Numb, a cell membrane associated protein previously studied in the context of asymmetric cell division and showed its requirement for establishment of PCP in the eye (P. Domingos, H. Steller and B. Mollereau, Rockefeller University, New York).

The Drosophila eye has also been used to study apical-basal polarity. The establishment of apical basal cell polarity is crucial for epithelial morphogenesis and development. Photoreceptors in the fly and higher animals are highly polarized as the light-sensing organs are formed at the most apical region of the cells. N.A. Bulgakova and E. Knust (Heinrich-Heine Universitaet, Dusseldorf, Germany) showed structure function analysis of DrosophilaStardust (Sdt) protein, a component of the Crumbs complex, which is one of the three protein complexes responsible for apical basal polarity in epithelia. Sdt contains two L27 domains and one SH3 domain. By generating transgenic flies expressing full length and altered forms of Sdt, they were able to show that proper localization of Sdt protein in the eye occurs by different mechanisms.

\section{Retinal development}

In the Pattern Formation session, there were some very interesting presentations on patterning using the Drosophilaeye model. Despite several different molecular genetic approaches, like the ability of a gene to induce ectopic eyes upon overexpression and the loss of eye in their mutant background, all efforts so far have been able to identify only seven genes controlling early retinal cell fate specification and determination viz., PAX6 homolog eyeless (ey), twin of eyeless (toy), eyegone (eyg), twin of eyegone (toe), eyesabsent (eya), sine oculis (so), dachshund (dac)and optix (opt)in Drosophila. These genes are referred to as retinal determination (RD) genes.

Justin Kumar's research group (Indiana University, Bloomington) presented their work on the initial developmental events that determine the fate of the fruit fly compound eye. Multiple signaling pathways are integrated into a complex regulatory network of nuclear factors to control the fate of the fly retina. First, they expressed the members of the RD pathway in over 200 unique expression patterns within the developing imaginal discs 
and have made a temporal and spatial map of the ability of each gene to induce eye development in non-retinal tissues. They were able to demonstrate that each gene has a unique ability to induce eye formation. Kumar et al. also conducted a series of genetic screens to identify upstream regulators of several current eye specification genes. It resulted in identification of multiple upstream regulators of dachshund and eyes absent and identified numerous genes that interact with sine oculis. The identification and functional characterization of these genes will provide us with a more complete picture of the regulatory network that governs the development of the eye.

Burnett et al. studied the physical interaction of Ey and Dac (encoded by members of the RD network) with Distal antenna (Dan) and Distal antenna related (Danr) protein. Dan and Danr proteins contain a Pipsqueak domain, a DNA binding motif implicated in chromatin modeling and have been found essential for proper specification of the Drosophilaeye. Burnett et al. suggested that Dan and Danr may form a complex with eye specification factors that may help regulate their activities. They have also extended their genetic interaction studies of dan and danr with other genes to discern their role during eye development [M.E. Burnett and J. Curtiss, (NMSU, Las Cruces, USA) and M. Mlodzik (Mount Sinai School of Medicine, New York, USA)].

\section{Signaling pathways}

Drosophila eye development involves the contribution of various signaling pathways. Among them, the receptor tyrosine kinase pathway, which involves activation of the Raf kinase by GTPbound Ras needs further understanding. A novel gene Aveugle was reported to be required for RAF activation in the Drosophila EGF receptor signaling pathway (J. Roignant and J. Treisman, Skirball Institute, New York University School of Medicine, NY, USA). Aveugleencodes a SAM domain protein that can physically interact with the scaffold protein, Connector enhancer of Ksr (Cnk) to promote Raf activation, perhaps by recruiting an activating kinase. A paper from Marc Therrien's group suggests that the gene Hyphen recruits $\mathrm{Ksr}$ to $\mathrm{Cnk}$ and that $\mathrm{Ksr}$ promotes an allosteric transition in Raf which allows it to autophosphorylate.

The Dpp pathway plays multiple roles that are essential for regulation of cell shape and patterning of the pupal retina. Cordero et al. demonstrated that Dpp has two separable roles during pupal eye development. First, Dpp is required for stable positioning of cells within the epithelium. Second, retinal cells require Dpp signaling to achieve their correct shape and position within the ommatidial hexagon (J.B. Cordero and R.L. Cagan, Washington University, St. Louis, USA).

The highly conserved JAK-STAT pathway regulates the size of the Drosophila eye. However, the mechanisms controlling growth are not fully understood. To elucidate the function of the JAK-STAT pathway, Bach's group reduced and increased functional levels of the transcription factor Stat92E in eye disc, which resulted in transcriptional activation and suppression, respectively, of wingless $(w g)$ in the eye disc. They found that Stat92E represses $w g$ expression in the eye. These studies may have an important bearing on early eye development, growth and patterning (L. Ekas and E. Bach, New York University School of Medicine, New York, USA).

Another interesting story was the demonstration of the role of

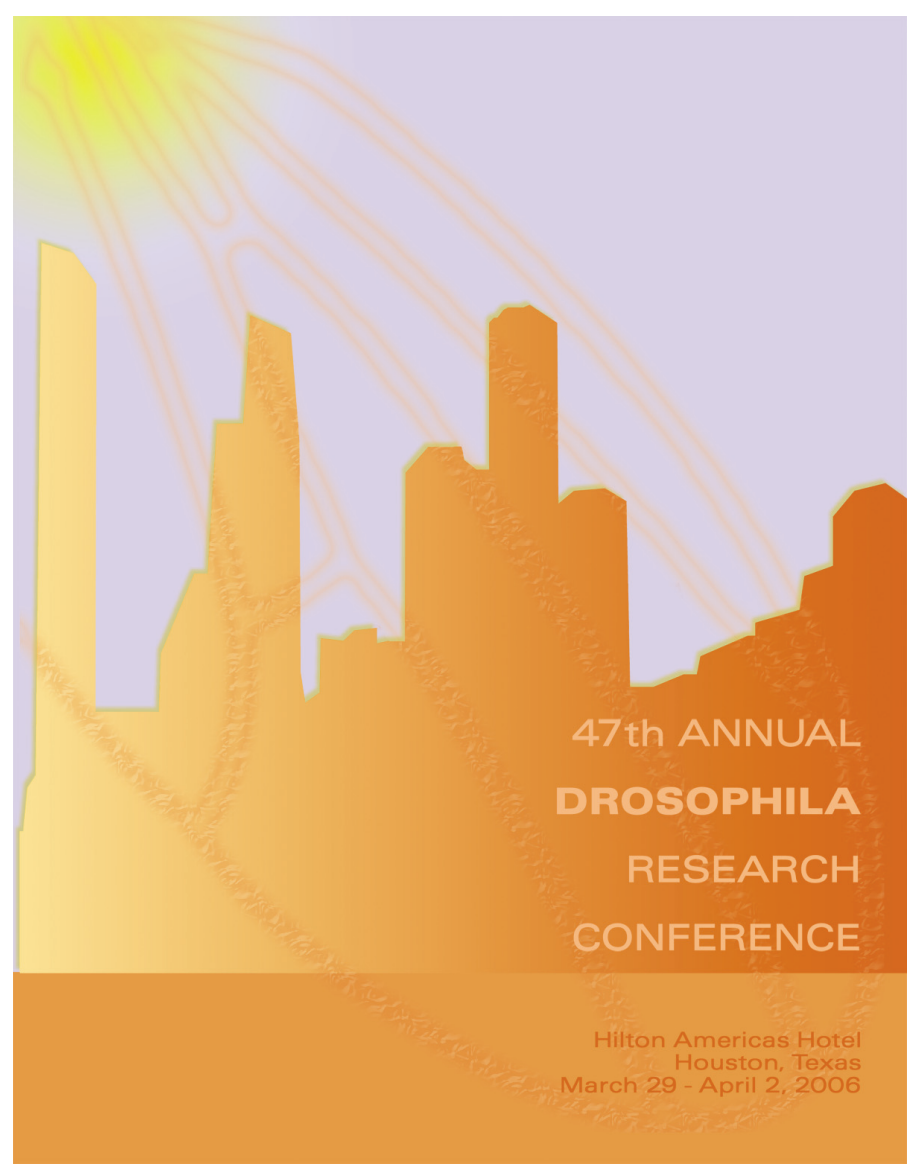

Poster of the Annual "Fly Meeting" held at Houston, Texas, USA from March 29th - April 2nd, 2006. Printed with permission from the Genetics Society of America.

Warts, a member of highly conserved Hippo/Salvador/Warts organ size control pathway in the regulation of rhodopsin expression in R8 photoreceptors. Jukam et al. showed that Warts regulates rhodopsin expression. They presented their genetic interaction analysis and preliminary model for how the warts arm of the bistable loop directs rhodopsin choice (D.Jukam, D. Pistillo and C. Desplan. New York University, New York).

\section{Early eye development and patterning}

Early eye development of Drosophila remains poorly understood. During early eye development, generation of dorsal-ventral (DV) patterning is the first lineage restriction event, which results in the generation of dorsal and ventral compartments. The border between the dorsal and ventral compartment is known as the equator and is the site of activation of $\mathrm{N}$ signaling. $\mathrm{N}$ signaling is known to play a crucial role in cell proliferation and differentiation of the developing eye field. Understanding the genetic basis of DV patterning in the eye is a very interesting problem. Andrew Tomlinson's group showed that sloppy-paired 1 and 2 (s/p 1 and $s / p 2$ ), functionally redundant genes encoding proteins of the forkhead transcription factor family, show domain-specific expression restricted to the ventral half of the eye. They also demonstrated that $s / p 1$ and s/p2 play an important role in DV 
patterning of the eye (A. Sato and A. Tomlinson, Columbia University, New York).

A. Singh and K. Choi (Baylor College of Medicine, Houston) presented a possible new role of members of Notch $(\mathrm{N})$ signaling pathway in cell survival during early eye development. During organogenesis there is an initial surge of cell proliferation leading to differentiation, which is followed by cell death to remove extra cells. Interestingly, there is little or no cell death during early development suggesting that survival mechanisms are in place. However, there is a gap in information about the genes required for survival during the early cell proliferation phase. Singh and Choi showed that Lobe and the Notch ligand Serrate (Ser), involved in early eye development and growth, are required for cell survival in the early eye disc. They presented that the loss of eye in Lobeand Sermutants is due to induction of cell death and upregulation of secreted Wingless $(\mathrm{Wg})$. They also presented evidence that Lobeand Ser loss-of-function trigger both caspasedependent and -independent cell death. These studies thus identify a mechanism responsible for cell survival in the early eye and a novel role of Notch signaling in cell survival.

The complex eye-antennal imaginal disc of Drosophila gives rise to the two separate adult organs: the adult eye and the antenna. A very important question being addressed by many research groups is how and when the decision of division of the complex eye-antennal disc to eye and antennal region takes places during development. A new transcription factor Dip3 from the MADF/BESS family was shown to be involved in the decisionmaking process of how a complex eye antennal disc is divided into eye and antennal field. Overexpression of Dip3 results in the duplication of antennal field and also in eye-to-antenna transformation. Dip3 is required in a cell-autonomous fashion for hedgehog pathway activation and in a non cell-autonomous fashion to regulate the retinal determination genes $(H$. Duong and $A$. Courey, UCLA, CA, USA; C.W. Wang and Y.H. Sun, Academia Sinica, Taipei, Taiwan).

\section{Screens}

The Drosophila eye model has also been exploited for many genome-wide and high throughput screens. Retinal cell fate determination is the early phase during fly eye development and only a handful of RD genes are known to control this process. To better understand the genetic network controlling this early process, Li and colleagues took a combinatorial approach of genetics, comparative genomics and computational biology to further identify potential direct downstream targets of the RD gene, eyeless (ey). They identified nearly 20 new putative direct targets of Ey and are now validating and characterizing such targets in vivo. Initial analysis revealed three genes eyes absent, shifted and Optix as novel targets of Ey (Y. Li, G. Mardon and R. Chen, Baylor College of Medicine, Houston, TX, USA).

There are many signaling pathways involved in cell fate determination in the Drosophila eye. Notch and EGFR signaling act antagonistically to each other. Notch signaling is required for Sphase re-entry of all cells in the second mitotic wave (SMW), whereas EGFR signaling prevents it by opposing Notch signaling. Firth and Baker employed a microarray based approach to identify the downstream targets by comparing gene expression profiles from two sets of eye discs: where SMW was present and absent due to the presence and absence of Notch. They have identified some promising candidates and their validation is in progress (L.C. Firth and N.E. Baker, Albert Einstein College, New York, USA).

Senseless (Sens), a conserved protein (PAG-3 in C.elegans and Gfi- 1 in mice and humans) plays an important role in neuronal cell development in the Drosophila eye. Sens is known to be necessary and sufficient for the development of R8 photoreceptor and intraommatidial bristles (IOBs). To identify new components of the senspathway and to discern the molecular mechanisms of sens function in the eye, Pepple et al. employed an F1 dominant modifier screen by driving overexpression of sens and screened for phenotypes in eye and the IOBs. Screening 110,000 flies, they were able to identify 117 suppressors and 11 enhancers that include some known and some novel genes. They are in the process of characterization of the various modifiers (K. Pepple and G. Mardon, Baylor College of Medicine, Houston, TX, USA).

G. Daftary and T. Xu (Yale university, New Haven, USA) used Drosophila eye for a genome-wide screen to gain insights into the genetic basis for tumor progression and metastasis. Overexpression of oncogenic Ras (RasV12) in the developing Drosophilaeye using the MARCM system results in non-invasive benign tumors. Introduction of mutations in the apico-basal polarity genes causes RasV12-induced benign tumors to exhibit metastatic behavior. Using the chemical mutagen EMS, they introduced additional mutations in the RasV12-induced benign tumors and screened these mutants for their ability to affect tumor growth or to cause tumor invasion to adjacent tissues such as the ventral nerve cord or leg discs or for metastasis to distant larval tissues. They identified many mutants (including cell polarity mutants) that exhibit invasive/metastatic behaviors and hundreds of mutants that dramatically promote or suppress tumor growth.

Drosophila is a highly versatile model for expressing foreign genes. A rich repository of tools to perform functional and genetic analysis are available for studies with Drosophila. Henry Sun's group has undertaken an interesting project to study human genes with unknown functions in Drosophila. Their approach is to ectopically express these novel human genes in Drosophilausing the GAL4/UAS system and observe the phenotypes caused by ectopic expression in different developmental stages. Using this approach they are in the process of generating a database of genes that show interesting phenotypes. The information generated from this exhaustive gain-of-function project will be extrapolated to higher vertebrate models $(\mathrm{Y}-\mathrm{H}$ Chang and $\mathrm{H}$. Sun, Academia Sinica, Taipei, Taiwan).

\section{Disease models}

Retinal degeneration is a common genetic disorder in humans and consists of a very diverse group of diseases. Dolph's lab presented a talk on "Light-dependent retinal degeneration in trafficking mutants of Drosophila". They presented their work on the model for Autosomal Dominant Retinitis Pigmentosa (ADRP) and the mechanism by which cell death is induced. They used the Drosophila eye as a model to study the mechanism of induction of cell death. In Drosophila, as in humans, mutations in the genes involved in the visual transduction pathway result in light-dependent retinal degeneration. ADRP, a genetic disorder, results in structural defects in cells, improper rhodopsin trafficking and 
abnormal phototransduction cascades, which result in photoreceptor cell apoptosis. The phototransduction pathway is shutoff by the binding of the abundant cytosolic molecule, arrestin, to rhodopsin. Using phospholipase $\mathrm{C}$ (norpA) eye mutant, they showed that endocytosis of stable rhodopsin-arrestin complex is responsible for the degeneration observed in norpA. These mutants that belong to the so called "granule group" of genes display retinal degeneration in a light-dependent manner. Further, these trafficking mutants disrupt transport to the lysosome (Y. Chinchore and P. Dolph, Biological Science, Dartmouth College, NH, USA).

Wallerian degeneration occurs in distal axons and synaptic terminals due to nerve injury. In slow wallerian degeneration (WIds) mutant mice, which have chimeric genes for Ube $4 \mathrm{~b} / \mathrm{Nmnat}$ (nocotinamide mononucleotide adenyltransferase), this degeneration process is delayed by 2-3 weeks. However, the mechanism is not fully understood. Hugo Bellen's group reported a fly model for NMNAT. They showed that mutation in NMNAT results in degeneration in photoreceptors, rhabdomeres, cell bodies and photoreceptor terminals in eye. They were able to rescue the NMNAT mutant phenotype by introducing wild-type NMNAT gene function. Furthermore, they demonstrated that NMNAT overexpression can protect Ataxin-1 induced neurodegeneration and may serve as a potent neuroprotective agent (R. Zhai and H.J. Bellen, Baylor College of Medicine, Houston, TX, USA).

\section{Community resources}

One of the benefits of working with Drosophila is the easy access to abundant research resources. In this meeting, the groups that are instrumental in generating and maintaining these resources for the fly community presented their work. A few of them are listed here.

\section{The Berkeley Drosophila Transcription Network Project}

(http://bdtnp.ilb.gov) is conducting a system-wide analysis of transcription factor networks in the early Drosophilaembryo. This multidisciplinary effort involves novel imaging, unique analysis and visualization methods to construct the first three-dimensional (3D) atlas of gene expression and morphology in an embryo at the cellular blastoderm stage.

\section{Flyexpress}

(http://www.flyexpress.net) is an image matching web tool for finding genes with overlapping expression patterns in Drosophila embryo. They have generated a database that includes photographs of Drosophila embryos showing 44,000 gene expression patterns for about 3,000 genes using gene-specific probes.

\section{The BDGP Gene Disruption Project}

(http://flypush.imgen.bcm.tmc.edu/pscreen). The aim of this project is to provide the community with a collection of transposition insertions affecting each Drosophila gene. Random insertions are generated with no bias for mutant phenotypes. The transposon insertion is localized by a flanking sequence. The useful insertions are sent to Bloomington stock center. This year the BDGP collection contains 7,140 mutant lines that provide access to at least 5,362 of the 13,666 annotated genes ( $A$. Spradling, Carnegie Institution, Baltimore, USA; R.A. Hoskins, Lawrence Berkeley National Laboratory, Berkeley, USA; H.J.
Bellen, Baylor College of Medicine, Houston, TX, USA).

\section{The Carnegie Protein Trap Collection}

Allan Spradling's lab at Carnegie Institution, Baltimore, USA, has constructed a large library of protein trap lines as a general source for determining the expression programs of Drosophila proteins, including their patterns of sub-cellular localization. They have at least 600 proteins trapped. (http://www.ciwemb.edu/labs/ spradling/buszczak/CIWProteinTrapLines.xls)

\section{Fly RNAi}

(http://flyrnai.org/). The completion of the genome sequences of a number of organisms has allowed comprehensive analysis of gene function on a genome-wide scale using RNA interference (RNAi). At the Drosophila RNAi Screening Center (DRSC), they are using a library directed against all predicted open reading frames in Drosophila. This library is available for the use of visiting scientists wishing to perform full-genome RNAi screens. The data generated from these screens are collected and archived in the DRSC database (http://flyRNAi.org/cgi-bin/RNAi_20 screens.pl). The long-term goal of this database is to provide annotations for as many of the uncharacterized genes in Drosophila as possible (N. Perrimon and B. Mathey-Prevot, Harvard Medical School Boston, USA and R. Paro, Heidelberg, Germany).

\section{Flybase}

FlyBase is an exhaustive database of genetic and molecular data for Drosophila (http://flybase.org/). FlyBase includes data on all species from the family Drosophilidae and can provide individual comparative analyses of the genomes of 12 species of the genus Drosophila. A common problem was that any search query would return information that was not specific or not relevant. In this meeting, the Flybase launched a trial version of its new interface, Query Builder (QB). QB provides a one-stop shop for information in FlyBase. QB presents a simple user interface that supports powerful searches due to its ability to include any combination of datasets in the same search.

\section{Conclusion}

The meeting turned out to be a great success in providing a platform for presenting recent advances in a wide spectrum of fly model system research. The local organizers, the fly community and the Genetics Society of America deserve the credit for the success of the meeting. This meeting further validated the point that model organism genetics holds immense promise for advancing biology, medicine and human genetics. I look forward to the next fly meeting at Philadelphia!

\section{Acknowledgements}

lextend my apology to those whose work / could not cite due to space constraint. I am thankful to the members of the fly community for their inputs. AS is supported by Retina Research Foundation, Fight for Sight Inc. and Knight's Templar Ophthalmology Research Foundation.

Received: 5th June 2006 Reviewed by Referees: 10th July 2006 Modified by Authors and Accepted for Publication: 16th August 2006 Published Online: 2nd October 2006 